Lyapunov exponents for linear delay equations in arbitrary phase spaces

Riedle, Markus

2005

MIMS EPrint: 2007.167

Manchester Institute for Mathematical Sciences

School of Mathematics

The University of Manchester

\footnotetext{
Reports available from: http://eprints.maths.manchester.ac.uk/

And by contacting: The MIMS Secretary

School of Mathematics

The University of Manchester

Manchester, M13 9PL, UK
} 


\title{
Lyapunov Exponents for Linear Delay Equations in Arbitrary Phase Spaces
}

\author{
Markus Riedle
}

\begin{abstract}
A linear integral equation with infinite delay is considered where the admissible function space $\mathcal{B}$ of initial conditions is as usually only described axiomatically. Merely using this axiomatic description, the long time behavior of the solutions is determined by calculating the Lyapunov exponents. The calculation is based on a representation of the solution in the second dual space of $\mathcal{B}$ and on a connection between the asymptotic behavior of the solutions of the integral equation under consideration and its adjoint equation subject to the spectral decomposition of the space of initial functions. We apply the result to an example of a stochastic differential equation with infinite delay.
\end{abstract}

Mathematics Subject Classification (2000). Primary 34K06; Secondary 34K50.

Keywords. Lyapunov exponents, differential equations with infinite delay, weak $^{*}$-integral, abstract phase space, variation of constants formula, stochastic differential equations with delay.

\section{Introduction}

For a function $x: \mathbb{R} \rightarrow \mathbb{C}^{d}$ with $d \in \mathbb{N}$ we define the history of $x$ for $t \geqslant 0$ by the function $x_{t}:(-\infty, 0] \rightarrow \mathbb{C}^{d}$ with $x_{t}(u):=x(t+u)$ for $u \leqslant 0$. We consider the following initial value problem

$$
\begin{aligned}
& x(t)=\varphi(0)+\int_{0}^{t} L\left(x_{s}\right) d s+h(t), \quad t \geqslant 0, \\
& x(u)=\varphi(u), \quad u \leqslant 0,
\end{aligned}
$$

where the initial condition $\varphi$ is an element of the so-called phase space $\mathcal{B}$. The space $\mathcal{B}$ is a linear subspace of the set of $\mathbb{C}^{d}$-valued functions on the negative real line equipped with a semi-norm $\|\cdot\|_{\mathcal{B}}$. We assume on $\mathcal{B}$ the topology induced by the semi-norm. The operator $L: \mathcal{B} \rightarrow \mathbb{C}^{d}$ is linear and continuous on $\mathcal{B}$. The function $h:[0, \infty) \rightarrow \mathbb{C}^{d}$ is continuous with $h(0)=0$. A solution of $(1.1)$ is a function 
$x: \mathbb{R} \rightarrow \mathbb{C}^{d}$ which is continuous on $[0, \infty)$ and satisfies the first relation in (1.1) with $x_{0}=\varphi$.

The history $x_{t}$ at time $t \geqslant 0$ of a solution $x$ of (1.1) always contains the initial data. Consequently, the choice of the space $\mathcal{B}$ is crucial and for every new space under consideration a new development of the theory would be required. To avoid this problem, an axiomatic description of the phase space has turned out to be an appropriate approach for differential equations with infinite delay, see [3] or $[5]$.

Only assuming the axiomatic description of the phase space $\mathcal{B}$, we calculate for a solution $x$ of equation (1.1) the Lyapunov exponents

$$
\lim _{t \rightarrow \infty} \frac{1}{t} \log \left\|x_{t}\right\|_{\mathcal{B}}
$$

or in case the limit (1.2) does not exist a bound for the upper limit point.

If there is a constant $\rho \geqslant 0$ such that the operator $L$ depends only on the function $\varphi$ restricted to the interval $[-\rho, 0]$ for every function $\varphi \in \mathcal{B}$ we say that (1.1) is of finite delay of length $\rho$.

If the delay is of finite length $\rho$ and the perturbation function $h$ is differentiable and if moreover the space of initial functions consists of continuous functions on the interval $[-\rho, 0]$ then a direct application of the spectral decomposition of the space of initial functions to the usual variation of constants formula for the solution yields the Lyapunov exponents. The most important estimates for that are summarized in Theorem 7.9.1 in [4]. But if the function $h$ is only assumed to be continuous and if the initial functions are measurable and bounded on the interval $[-\rho, 0]$ the calculation of the Lyapunov exponents requires already more efforts. The result in this case of an equation with finite delay is achieved in [9] and [10]. Their approach is still based on the spectral decomposition of the space of initial functions and the variation of constants formula in the same space but also depends strongly on properties of equations with finite delay and on the specific choice of the space of initial functions.

In the more general case in this paper of infinite delay and of axiomatically described phase spaces such a direct calculation of the Lyapunov exponents fails. But we are able to calculate the Lyapunov exponents by a new method which we describe by the following steps:

1. representation of the history $x_{t}$ by $x_{t}^{* *}$ in the second dual space $\mathcal{B}^{* *}$

2. spectral decomposition of $\mathcal{B}$ by $\mathcal{B}=P \oplus Q$, where $P$ is a finite dimensional subspace

3 . determine the asymptotic of $\pi_{P}^{* *} x_{t}^{* *}$ where $\pi_{P}$ denotes the projection onto $P$ and $\pi_{P}^{* *}$ denotes the second adjoint operator

4. determine the asymptotic of $\pi_{Q}^{* *} x_{t}^{* *}$ where $\pi_{Q}$ denotes the projection onto $Q$ and $\pi_{Q}^{* *}$ denotes the second adjoint operator

5. summarizing the estimates of $\pi_{P}^{* *} x_{t}^{* *}$ and $\pi_{Q}^{* *} x_{t}^{* *}$ to obtain the Lyapunov exponents 
For the representation in the bidual space under 1 we introduce a modified weak*integral whereas the decomposition of $\mathcal{B}$ is a well-known result for linear differential equations. The asymptotic under 3 can be determined due to the finite dimensionality of $P$. We succeed in estimating the asymptotic in 4 by means of the long time behavior of the solution of the so-called adjoint equation of (1.1). The main idea for this estimate is to consider the adjoint equation but with respect to the decomposition under 2 in some sense. By this method we demonstrate the usefulness of the adjoint equation not only for obtaining a variation of constants formula in the bidual space.

We begin in section 2 with recalling some basics on differential equations with infinite delay. For details as well for motivation of the axiomatic description of the phase space $\mathcal{B}$ we refer the reader to the monograph [5]. In section 3 we represent the history of the solution in the second dual space of the phase space $\mathcal{B}$. In section 4 we determine the long time behavior of the two projections mentioned above under 3 and 4 (Theorem 4.2 and 4.4). By summarizing these results we obtain in Theorem 4.5 the Lyapunov exponents. In section 5 we introduce an example of a stochastic differential equation with infinite delay and we apply the result on the Lyapunov exponents to this equation. Due to the usual assumptions in the frame work of stochastic differential equations the set of Lyapunov exponents turns out to contain only one element.

\section{Linear Autonomous Systems}

We consider linear autonomous differential equations with infinite delay:

$$
\dot{x}(t)=L\left(x_{t}\right) \text { for a.e. } t \geqslant 0, \quad x_{0}=\varphi \in \mathcal{B} .
$$

We say, that a solution of $(2.1)$ is a function $x=x(\cdot, \varphi)$ on $\mathbb{R}$ which is locally absolutely continuous on $[0, \infty)$ and satisfies the first equation in $(2.1)$ with $x_{0}=\varphi$.

The space $\mathcal{B}=\mathcal{B}\left((-\infty, 0], \mathbb{C}^{d}\right)$ is always assumed to be a linear subspace of $\left\{\varphi:(-\infty, 0] \rightarrow \mathbb{C}^{d}\right\}$ with semi-norm $\|\cdot\|_{\mathcal{B}}$. A norm on $\mathbb{C}^{d}$ is denoted by $|\cdot|$. We denote by $C\left(J, \mathbb{C}^{d}\right)$ the space of bounded continuous functions mapping an interval $J$ into $\mathbb{C}^{d}$ with the norm $\|f\|_{C(J)}:=\sup \{|f(u)|: u \in J\}$. We denote by

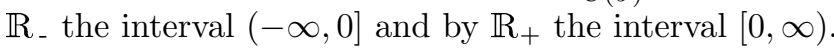

In the sequel we summarize the conditions on $\mathcal{B}$ as they are proposed in [5].

Condition (A). For every function $x: \mathbb{R} \rightarrow \mathbb{C}^{d}$ which is continuous on $[0, \infty)$ and satisfies $x_{0} \in \mathcal{B}$ the following conditions hold for every $t \geqslant 0$ :

1) $x_{t} \in \mathcal{B}$

2) there exists $H>0$, independent of $x$ and $t$, such that $|x(t)| \leqslant H\left\|x_{t}\right\|_{\mathcal{B}}$;

3 ) there exists $N:[0, \infty) \rightarrow[0, \infty)$, continuous, independent of $x$ and $t$, there exists $M:[0, \infty) \rightarrow[0, \infty)$, locally bounded, independent of $x$ and $t$, such 
that:

$$
\left\|x_{t}\right\|_{\mathcal{B}} \leqslant N(t) \sup _{0 \leqslant u \leqslant t}|x(u)|+M(t)\left\|x_{0}\right\|_{\mathcal{B}} .
$$

4) $t \mapsto x_{t}$ is a $\mathcal{B}$-valued continuous function.

A space $\mathcal{B}$ satisfying Condition $(\mathrm{A})$ always contains the space $C_{c}\left(\mathbb{R}_{-}, \mathbb{C}^{d}\right)$,

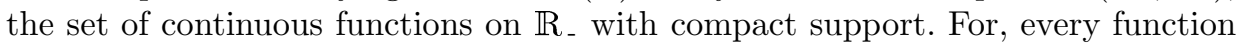
$\varphi$ which is continuous on $\mathbb{R}_{+}$and vanishes on $\mathbb{R}_{\text {- }}$ is a function considered in Condition (A.1).

In [5] it is shown that the homogeneous equation (2.1) has a unique solution under Condition (A), if the operator $L$ is linear and bounded.

For $\varphi \in \mathcal{B}$ the symbol $\hat{\varphi}$ denotes the equivalence class $\left\{\psi \in \mathcal{B}:\|\psi-\varphi\|_{\mathcal{B}}=\right.$ $0\}$. The quotient space $\hat{\mathcal{B}}:=\mathcal{B} /\|\cdot\|_{\mathcal{B}}$ is a linear space with norm $\|\hat{\varphi}\|_{\hat{\mathcal{B}}}=\|\varphi\|_{\mathcal{B}}$. For a bounded linear operator $T$ on $\mathcal{B}$ let $\hat{T}$ be the induced operator $\hat{T} \hat{\varphi}=T \varphi$ for some $\varphi \in \hat{\varphi}$.

Condition (B). The quotient space $\hat{\mathcal{B}}$ is complete.

Condition (C). For every Cauchy sequence $\left\{\varphi_{n}\right\} \subseteq \mathcal{B}$ with respect to the seminorm $\|\cdot\|_{\mathcal{B}}$, which converges uniformly on every compact subset of $\mathbb{R}$. to a function $\varphi$, the function $\varphi$ is in $\mathcal{B}$ and $\left\|\varphi_{n}-\varphi\right\|_{\mathcal{B}}$ tends to zero for $n \rightarrow \infty$.

We present two examples of function spaces satisfying the Conditions (A), (B) and (C). For details we refer to [5].

Example. For $\gamma \in \mathbb{R}$ define

$$
\begin{aligned}
C_{\gamma}\left(\mathbb{R}_{-}, \mathbb{C}^{d}\right) & :=\left\{\varphi \in C\left(\mathbb{R}_{-}, \mathbb{C}^{d}\right): \lim _{u \rightarrow-\infty}|\varphi(u)| e^{-\gamma u} \text { exists in }[0, \infty)\right\}, \\
\|\varphi\|_{C_{\gamma}} & :=\sup _{u \leqslant 0}\left|\varphi(u) e^{-\gamma u}\right| .
\end{aligned}
$$

The space $C_{\gamma}\left(\mathbb{R}_{-}, \mathbb{C}^{d}\right)$ satisfies the Conditions (A), (B) and (C), where the functions $N$ and $M$ in (A) can be chosen as

$$
N(t)=\max \left\{1, e^{\gamma t}\right\} \quad \text { and } \quad M(t)=e^{\gamma t} \text { for } t \geqslant 0 .
$$

Example. For a nonnegative locally integrable function $g: \mathbb{R}_{-} \rightarrow[0, \infty)$ and $p \geqslant 1$ define

$$
\begin{aligned}
\left(\mathbb{C}^{d} \times L_{g}^{p}\right)\left(\mathbb{R}_{-}, \mathbb{C}^{d}\right) & :=\left\{\varphi: \mathbb{R}_{-} \rightarrow \mathbb{C}^{d}: \int_{-\infty}^{0}|\varphi(u)|^{p} g(u) d u<\infty\right\} \\
\|\varphi\|_{\mathbb{C}^{d} \times L_{g}^{p}} & :=|\varphi(0)|+\left(\int_{-\infty}^{0}|\varphi(u)|^{p} g(u) d u\right)^{1 / p}
\end{aligned}
$$

If there exists a locally bounded function $G: \mathbb{R}_{-} \rightarrow[0, \infty)$ such that $g(u+s) \leqslant$

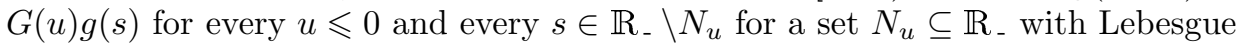


measure 0 then the space $\left(\mathbb{C}^{d} \times L_{g}^{p}\right)\left(\mathbb{R}_{-}, \mathbb{C}^{d}\right)$ satisfies the Conditions (A), (B) and (C). The functions $N$ and $M$ in Condition (A) can be chosen as

$$
N(t)=1+\left(\int_{-t}^{0} g(u) d u\right)^{1 / p}, \quad M(t)=\max \left\{\left(\int_{-t}^{0} g(u) d u\right)^{1 / p}, G(-t)^{1 / p}\right\}
$$

for $t \geqslant 0$.

This example of a space of initial functions for differential equations with infinite delay often occurs in studies of mechanics of materials with memory, see [7] and the references therein.

In the sequel suppose that the phase space $\mathcal{B}$ satisfies Condition (A) and that $L$ is a linear bounded operator from $\mathcal{B}$ into $\mathbb{C}^{d}$. For an arbitrary linear bounded operator $T: X \rightarrow Y$ and semi-normed linear spaces $X$ and $Y$, we denote the operator norm of $T$ by $\|T\|_{X \rightarrow Y}$. We call a function $f$ on $(-\infty, 0]$ of bounded variation normalized if it is left continuous on $(-\infty, 0)$ and $f(0)=0$.

Based on the Riesz representation theorem one obtains the following theorem which is proved in Theorem 3.4.2 in [5].

Theorem 2.1. For every linear bounded operator $L: \mathcal{B} \rightarrow \mathbb{C}^{d}$ there exists a unique function $\mu:(-\infty, 0] \rightarrow \mathbb{C}^{d \times d}$ locally of bounded variation and normalized with

$$
\begin{aligned}
& L \varphi=\int d \mu(u) \varphi(u) \quad \text { for all } \varphi \in C_{c}\left(\mathbb{R}_{-}, \mathbb{C}^{d}\right), \\
& \operatorname{Var}\left(\mu,\left[a_{1}, a_{2}\right]\right) \leqslant c\|L\|_{\mathcal{B} \rightarrow \mathbb{C}^{d}} N\left(a_{2}-a_{1}\right) M\left(-a_{2}\right) \quad \text { for } a_{1}<a_{2} \leqslant 0,
\end{aligned}
$$

where $\operatorname{Var}(f, J)$ denotes the total variation of a function $f$ on the interval $J$ and $c$ is a constant depending on the norm of $\mathbb{C}^{d}$.

The function $\mu$ according to Theorem 2.1 determines the fundamental equation of equation (2.1):

$$
\dot{r}(t)=\int_{[-t, 0]} d \mu(u) r(t+u) \quad \text { for almost every } t \geqslant 0, \quad r(0)=\mathrm{I},
$$

where I denotes the identity matrix in $\mathbb{C}^{d \times d}$. Theorem 4.1.3 in [5] guarantees the existence of a unique locally absolutely continuous function $r:[0, \infty) \rightarrow \mathbb{C}^{d \times d}$ satisfying equation (2.4).

Let $\mathbb{C}^{d *}$ be the space of d-dimensional row-vectors. The function $\mu$ in Theorem 2.1 defines also the so-called adjoint equation of (2.1):

$$
y(s)+\int_{s}^{0} y(u) \mu(s-u) d u=b(s), \quad s \leqslant 0,
$$

with the forcing function $b: \mathbb{R}_{-} \rightarrow \mathbb{C}^{d *}$ being locally of bounded variation. According to Theorem 4.1.4 in [5] equation (2.5) has a unique solution $y=y(\cdot, b)$, which is locally of bounded variation for $s \leqslant 0$ :

$$
\operatorname{Var}(y,[s, 0]) \leqslant \operatorname{Var}(b,[s, 0])+\left(e^{-c s\|L\|_{\mathcal{B} \rightarrow \mathbb{C}^{d}}\|N\|_{C[0,-s]}}-1\right) \sup _{s \leqslant u \leqslant 0}|b(u)|
$$


with a constant $c>0$ depending on the norm of $\mathbb{C}^{d}$. If the forcing function $b$ is normalized, then so is the solution $y(\cdot, b)$. Furthermore, by Corollary 4.1.7 in [5] the solution of (2.5) is given by

$$
y(s)=b(0) r(-s)-\int_{[s, 0]} d b(u) r(u-s), \quad s \leqslant 0,
$$

where $r$ is the solution of (2.4).

Let $\mathcal{B}^{*}$ and $\hat{\mathcal{B}}^{*}$ be the dual spaces of $\mathcal{B}$ and $\hat{\mathcal{B}}$, respectively, which are Banach spaces with the usual operator norms, see [2]. We denote by $\langle\psi, \varphi\rangle$ the duality pairing of $\psi \in \mathcal{B}^{*}$ and $\varphi \in \mathcal{B}$. $\mathcal{B}^{*}$ is isometrically isomorphic to $\hat{\mathcal{B}}^{*}$ by the mapping $\varphi^{*} \mapsto \hat{\varphi}^{*}$ for $\varphi^{*} \in \mathcal{B}^{*}$, where $\hat{\varphi}^{*} \in \hat{\mathcal{B}}^{*}$ is defined by $\left\langle\hat{\varphi}^{*}, \hat{\varphi}\right\rangle=\left\langle\varphi^{*}, \varphi\right\rangle$ for every $\varphi \in \mathcal{B}$. In the same way one can identify the adjoint operator $\hat{T}^{*}$ of $\hat{T}$ with the adjoint operator $T^{*}$ of $T$ for a bounded linear operator $T$ on $\mathcal{B}$.

Theorem 2.1 implies that for every $\psi \in \mathcal{B}^{*}$ a unique $\widetilde{\psi}: \mathbb{R}_{-} \rightarrow \mathbb{C}^{d *}$ exists which is locally of bounded variation and normalized such that

$$
\begin{aligned}
& \qquad\langle\psi, \varphi\rangle=\int d \widetilde{\psi}(u) \varphi(u) \quad \text { for every } \varphi \in C_{c}\left(\mathbb{R}_{-}, \mathbb{C}^{d}\right), \\
& \text { and } \operatorname{Var}(\widetilde{\psi},[-t, 0]) \leqslant c^{\prime} N(t)\|\psi\|_{\mathcal{B}^{*}} \quad \text { for } t \geqslant 0,
\end{aligned}
$$

with a constant $c^{\prime}$ depending on the norm on $\mathbb{C}^{d}$. For $\psi \in \mathcal{B}^{*}$ we will always use the notation $\widetilde{\psi}$ or $\psi^{\widetilde{f}}$ for the transformation introduced above.

The solution of the homogeneous equation (2.1) and the solution of the adjoint equation (2.5) are related in the following way. Define the solution operators of the initial value problem (2.1) for $t \geqslant 0$ by

$$
T(t): \mathcal{B} \rightarrow \mathcal{B}, \quad T(t) \varphi=x_{t},
$$

where $x=x(\cdot, \varphi)$ is the solution of equation (2.1). Condition (A) implies that the operators $T(t)$ are continuous for every $t \geqslant 0$, for details see [5].

Then the adjoint operators $\left\{T^{*}(t)\right\}_{t \geqslant 0}$ of the solution operators $\{T(t)\}_{t \geqslant 0}$ and the solution $y$ of the adjoint equation (2.5) obey the equality

$$
\left[T^{*}(t) \psi\right]^{\sim}(0-)=y(-t, \widetilde{\psi}) \text { for every } t>0 \text { and } \quad \psi \in \mathcal{B}^{*} .
$$

A proof is given in Theorem 4.2.2 in [5].

\section{Representation in the second dual space}

Denote by $B V\left([a, b], \mathbb{C}^{k \times l}\right), k, l \in \mathbb{N}$, the space of functions of bounded variation mapping the interval $[a, b]$ into $\mathbb{C}^{k \times l}$. Equipped with the norm $\|f\|_{B V[a, b]}:=$ $|f(a)|+\operatorname{Var}(f,[a, b])$, it is a Banach space. 
Recall that for functions $f \in B V\left([a, b], \mathbb{C}^{k \times l}\right)$ and $h \in C\left([a, b], \mathbb{C}^{l}\right)$ the integral on the left hand side of

$$
\int_{[a, b]} f(s) d h(s)=-\int_{[a, b]} d f(s) h(s)+f(b) h(b)-f(a) h(a)
$$

is well defined by the right hand side, where the integral is understood as a Riemann-Stieltjes integral.

Let $\mu$ be the function corresponding to the operator $L$ of equation (1.1) according to Theorem 2.1 and let $r$ denote the solution of equation (2.4) with the function $\mu$. We define for $t \geqslant 0$ the operator

$$
\begin{aligned}
K(t): C\left([0, t], \mathbb{C}^{d}\right) & \rightarrow \mathcal{B}, \\
(K(t) h)(u) & := \begin{cases}\int_{0}^{t+u} r(t-s+u) d h(s), & u \in[-t, 0], \\
0, & u<-t .\end{cases}
\end{aligned}
$$

Since $K(t) h$ is a continuous function with compact support, it is an element of the phase space $\mathcal{B}$. By use of Gronwall's inequality and the Condition (A) one establishes that for all $t \geqslant 0$ the operator $K(t)$ is linear and bounded on $C\left([0, t], \mathbb{C}^{d}\right)$.

By means of the representation $(2.2)$ of $L$ on $C_{c}\left(\mathbb{R}_{-}, \mathbb{C}^{d}\right)$ one can derive (see [11]) that for every $\varphi \in \mathcal{B}$ and $h \in C\left(\mathbb{R}_{+}, \mathbb{C}^{d}\right)$ with $h(0)=0$ a unique solution $x=x(\cdot, \varphi, h)$ of equation (1.1) exists which is given in the phase space $\mathcal{B}$ by

$$
x_{t}=T(t) \varphi+K(t) h \text { for } t \geqslant 0 .
$$

Evaluating $x_{t}$ in zero yields the solution in the state space $\mathbb{C}^{d}$ at time $t$.

In order to determine the Lyapunov exponents (1.2) there seems not to be a direct way by use of (3.3) since estimating $\|K(t) h\|_{\mathcal{B}}$ for $t \rightarrow \infty$ results in estimating the matrix valued integrand $r$ in the definition (3.2) which is not related in any way to the phase space $\mathcal{B}$. We avoid this problem by embedding the $\mathcal{B}-$ valued representation (3.3) of the solution into the larger bidual space $\mathcal{B}^{* *}$. For that we introduce a weak*-integral in the dual space of a Banach space, which corresponds to the integral (3.1) in a similar way as the usual weak*-integral to the Lebesgue integral. Let $X$ be an arbitrary Banach space with the norm $\|\cdot\|_{X}$ and denote by $\left\langle x^{*}, x\right\rangle$ the dual pairing of $x^{*} \in X^{*}$ and $x \in X$.

Definition 3.1. A function $f:[a, b] \rightarrow X^{*}$ is called (Riemann-Stieltjes) weak*integrable on $[a, b]$ with respect to continuous functions if

1. the function $t \mapsto\langle f(t), x\rangle$ is of bounded variation on $[a, b]$ for each $x \in X$;

2. the linear operator

$$
F: X \rightarrow B V([a, b], \mathbb{C}), \quad F(x)(s):=\langle f(s), x\rangle, \quad s \in[a, b],
$$

is continuous.

Lemma 3.2. Let $f:[a, b] \rightarrow X^{*}$ be a weak ${ }^{*}$-integrable function with respect to continuous functions and $h \in C([a, b], \mathbb{C})$. Then there exists a unique element 
$x^{*} \in X^{*}$ such that

$$
\left\langle x^{*}, x\right\rangle=\int_{a}^{b}\langle f(s), x\rangle d h(s) \quad \text { for all } x \in X .
$$

Proof. Define the operator $F: X \rightarrow B V([a, b], \mathbb{C})$ as in Definition 3.1. Since the operator $F$ is assumed to be continuous, one obtains

$$
\begin{aligned}
\left|\int_{a}^{b}\langle f(s), x\rangle d h(s)\right| & \leqslant 2\|h\|_{C[a, b]}\|F(x)\|_{B V[a, b]} \\
& \leqslant 2\|h\|_{C[a, b]}\|F\|_{X \rightarrow B V}\|x\|_{X} .
\end{aligned}
$$

Hence, the linear functional $x \mapsto \int_{a}^{b}\langle f(s), x\rangle d h(s)$ is bounded and is thus an element of $\mathcal{B}^{*}$.

Lemma 3.2 allows to define a weak*-integral with respect to a continuous function.

Definition 3.3. Let $f:[a, b] \rightarrow X^{*}$ be a weak*-integrable function with respect to continuous functions and $h \in C([a, b], \mathbb{C})$. We define the weak ${ }^{*}$-integral of $f$ with respect to $h$ by the functional

$$
* \int_{a}^{b} f(s) d h(s) \in X^{*}: \quad\left\langle * \int_{a}^{b} f(s) d h(s), x\right\rangle:=\int_{a}^{b}\langle f(s), x\rangle d h(s)
$$

for all $x \in X$.

For $f=\left(f_{1}, \ldots, f_{d}\right)$ with $f_{i}:[a, b] \rightarrow X^{*}$ weak*-integrable with respect to continuous functions and $h \in C\left([a, b], \mathbb{C}^{d}\right)$ the weak*-integral $* \int f d h$ is defined component-wise.

The weak*-integral commutes in the following sense. Let $f:[a, b] \rightarrow X^{*}$ be a weak*-integrable function according to Definition 3.1 and $U: X \rightarrow X$ be a bounded linear operator. Then $U^{*} f$ is weak*-integrable with respect to continuous functions and

$$
U^{*} * \int_{a}^{b} f(s) d h(s)=* \int_{a}^{b} U^{*} f(s) d h(s) \quad \text { for every } h \in C([a, b], \mathbb{C}) .
$$

This can be shown analogously to the same property of the usual weak*-integral, see Lemma 3.14 in the Appendix II of [1].

We begin the introduction of the representation for the solution in the second dual space with a relation of the operators $K(t)$ and the adjoint operators $\left\{T^{*}(t)\right\}_{t \geqslant 0}$ of the solution operators $\{T(t)\}_{t \geqslant 0}$ in the following lemma.

Lemma 3.4. For $\psi \in \mathcal{B}^{*}$ and $h \in C\left([0, t], \mathbb{C}^{d}\right)$ we have

$$
\langle\psi, K(t) h\rangle=-\int_{0}^{t}\left[T^{*}(t-s) \psi\right]^{\sim}(0-) d h(s) \text { for every } t \geqslant 0 .
$$


Proof. The integral is well defined because $\left[T^{*}(t-s) \psi\right]^{\sim}(0-)=y(s-t, \widetilde{\psi})$ holds by $(2.10)$ and the solution $y(\cdot, \widetilde{\psi})$ of equation $(2.5)$ is locally of bounded variation. First, we assume that $h$ is a continuously differentiable function on $[0, t]$. Recall that $K(t) h$ is an element of $C_{c}\left(\mathbb{R}_{-}, \mathbb{C}^{d}\right)$ and $\widetilde{\psi}(0)=0$. By $(2.8),(2.7)$ and $(2.10)$ one obtains for every $\psi \in \mathcal{B}^{*}$

$$
\begin{aligned}
\langle\psi, K(t) h\rangle & =\int_{[-t, 0]} d \widetilde{\psi}(u)[K(t) h](u) \\
& =\int_{[-t, 0]} d \widetilde{\psi}(u)\left(\int_{0}^{t+u} r(t-s+u) d h(s)\right) \\
& =\int_{[-t, 0]} d \widetilde{\psi}(u)\left(\int_{0}^{t+u} r(t-s+u) \dot{h}(s) d s\right) \\
& =\int_{0}^{t}\left(\int_{[s-t, 0]} d \widetilde{\psi}(u) r(t-s+u)\right) \dot{h}(s) d s \\
& =-\int_{0}^{t} y(s-t, \widetilde{\psi}) \dot{h}(s) d s \\
& =-\int_{0}^{t}\left[T^{*}(t-s) \psi\right]^{\sim}(0-) d h(s) .
\end{aligned}
$$

As both sides of the equation depend continuously on $h$ with respect to the supremum norm and the continuously differentiable functions are dense in $C\left([0, t], \mathbb{C}^{d}\right)$ the assertion follows.

Define for $i=1, \ldots, d$ the functionals

$$
\gamma^{i}: \mathcal{B}^{*} \rightarrow \mathbb{C}, \quad \gamma^{i}(\psi):=-\widetilde{\psi}^{i}(0-)
$$

where $\widetilde{\psi}^{i}$ denotes the i-th component of $\widetilde{\psi}$ for $\psi \in \mathcal{B}^{*}$. We infer from the estimate $(2.9)$ and $\widetilde{\psi}(0)=0$

$$
\left|\gamma^{i}(\psi)\right|=\left|\widetilde{\psi}^{i}(0-)\right| \leqslant \operatorname{Var}(\widetilde{\psi},[-1,0]) \leqslant c^{\prime} N(1)\|\psi\|_{B^{*}} .
$$

Therefore we have $\gamma^{i} \in \mathcal{B}^{* *}$ for $i=1, \ldots, d$. We define $\gamma: \mathcal{B}^{*} \rightarrow \mathbb{C}^{d^{*}}$ by $\gamma(\psi):=\left(\gamma^{1}(\psi), \ldots, \gamma^{d}(\psi)\right)$, thus $\gamma(\psi)=-\tilde{\psi}(0-)$. Furthermore, we set $U \gamma:=$ $\left(U \gamma^{1}, \ldots, U \gamma^{d}\right)$ for an operator $U$ on $\mathcal{B}^{* *}$.

In the sequel we identify the space $\hat{\mathcal{B}}$ with the subspace of the second dual space $\mathcal{B}^{* *}$ in the usual manner. The dual pairing of $\psi \in \mathcal{B}^{*}$ and $\varphi^{* *} \in \mathcal{B}^{* *}$ is denoted by $\left\langle\psi, \varphi^{* *}\right\rangle$.

Now we replace the Banach space $X$ in Definition 3.3 of the weak*-integral by the dual space $\mathcal{B}^{*}$ to define a weak*-integral. By use of this weak*-integral we represent the history of the solution of equation (1.1) in the second dual space $B^{* *}$. A major part of calculating the Lyapunov exponents will be based on this representation. 
Theorem 3.5. Let $x=x(\cdot, \varphi, h)$ be the solution of $(1.1)$. Then $T^{* *}(t-\cdot) \gamma^{i}:[0, t] \rightarrow$ $\mathcal{B}^{* *}$ is weak ${ }^{*}$-integrable with respect to continuous functions for all $i=1, \ldots, d$ and

$$
\hat{x}_{t}=\hat{T}(t) \hat{\varphi}+* \int_{0}^{t} T^{* *}(t-s) \gamma d h(s) \quad \text { for } t \geqslant 0 .
$$

Proof. For $\psi \in \mathcal{B}^{*}$ we denote by $y^{i}=y^{i}(\cdot, \widetilde{\psi})$ the $\mathrm{i}$-th component of the solution $y(\cdot, \widetilde{\psi})$ of the adjoint equation (2.5). We obtain by (3.5) and $(2.10)$ for $s \in[0, t]$

$$
\left\langle\psi, T^{* *}(t-s) \gamma^{i}\right\rangle=\left\langle T^{*}(t-s) \psi, \gamma^{i}\right\rangle=-y^{i}(s-t, \widetilde{\psi}) .
$$

Consequently, the function $s \mapsto\left\langle\psi, T^{* *}(t-s) \gamma^{i}\right\rangle$ is of bounded variation on $[0, t]$ since $y(\cdot, \widetilde{\psi})$ is of bounded variation. By use of relation (3.7) the equations $(2.6)$ and (2.9) yield

$$
\begin{aligned}
\left\|\left\langle\psi, T^{* *}(t-\cdot) \gamma^{i}\right\rangle\right\|_{B V[0, t]} & =\left\|y^{i}(\cdot-t, \widetilde{\psi})\right\|_{B V[0, t]} \\
& \leqslant\|y(\cdot, \widetilde{\psi})\|_{B V[-t, 0]} \\
& =|y(-t, \widetilde{\psi})|+\operatorname{Var}(y(\cdot, \widetilde{\psi}),[-t, 0]) \\
& \leqslant 2\left(\operatorname{Var}(\widetilde{\psi},[-t, 0])+k(t) \sup _{-t \leqslant u \leqslant 0}|\widetilde{\psi}(u)|\right) \\
& \leqslant 2(1+2 k(t)) \operatorname{Var}(\widetilde{\psi},[-t, 0]) \\
& \leqslant 2(1+2 k(t)) c^{\prime} N(t)\|\psi\|_{\mathcal{B}^{*}},
\end{aligned}
$$

with $k(t):=\left(e^{c t\|L\|_{\mathcal{B} \rightarrow \mathbb{C}^{d}}\|N\|_{C[0, t]}}-1\right)$ and constants $c, c^{\prime}>0$ depending on the norm of $\mathbb{C}^{d}$. Hence, the function $s \mapsto T^{* *}(t-s) \gamma^{i}$ is weak*-integrable with respect to continuous functions for $i=1, \ldots, d$. Moreover, Lemma 3.4 implies

$$
\begin{aligned}
\left\langle\psi, * \int_{0}^{t} T^{* *}(t-s) \gamma d h(s)\right\rangle & =\int_{0}^{t}\left\langle\psi, T^{* *}(t-s) \gamma\right\rangle d h(s) \\
& =\int_{0}^{t}\left\langle T^{*}(t-s) \psi, \gamma\right\rangle d h(s) \\
& =-\int_{0}^{t}\left[T^{*}(t-s) \psi\right]^{\sim}(0-) d h(s) \\
& =\langle\psi, K(t) h\rangle .
\end{aligned}
$$

By means of (3.3) the assertion follows.

In contrast to the representation (3.3) of the solution of (1.1) the representation (3.6) allows an estimate of $\left\|\hat{x}_{t}\right\|_{\mathcal{B}}$ in the next section due to the larger space $\mathcal{B}^{* *}$. In particular, a part of this estimation will be based on the relation $(2.10)$ of the adjoint operators $T^{*}(t)$ and the solution $y(t, \cdot)$ of the adjoint equation $(2.5)$. 


\section{Estimates on the subspaces and the Lyapunov exponents}

First, we report on a result concerning a decomposition of $\mathcal{B}$ as it is stated in Chapter 5 of [5]. Assume in the sequel that the phase space $\mathcal{B}$ satisfies the Conditions $(\mathrm{A}),(\mathrm{B})$ and $(\mathrm{C})$ and that the operator $L$ is bounded on $\mathcal{B}$. From Condition (A), the solution operators $\{\hat{T}(t)\}_{t \geqslant 0}$ of equation (2.1) form a strongly continuous semi-group of bounded operators on $\hat{\mathcal{B}}$. Denote the generator of $\{\hat{T}(t)\}_{t \geqslant 0}$ by $\hat{A}$. The solution operators for equation (2.1) with the trivial operator $L=0$ are given by

$$
S(t): \mathcal{B} \rightarrow \mathcal{B}, \quad(S(t) \varphi)(u)= \begin{cases}\varphi(0), & u \in[-t, 0] \\ \varphi(t+u), & u \leqslant-t,\end{cases}
$$

for $t \geqslant 0$. Define a parameter $\beta$, depending only on the phase space $\mathcal{B}$, by the following relation

$$
\beta:=\lim _{t \rightarrow \infty} \frac{\log \alpha(\hat{S}(t))}{t},
$$

where $\alpha$ is the Kuratowski measure of non-compactness of bounded operators, see e.g. Definition 4.20 in the Appendix II of [1]. For a fixed $\lambda \in \mathbb{C}$ define the function $(e(\lambda) b)(u):=e^{\lambda u} b$ for $u \leqslant 0$ and an arbitrary $b \in \mathbb{C}^{d}$. If $\operatorname{Re} \lambda>\beta$ then the functions $e(\lambda) b$ are elements of $\mathcal{B}$ for every $b \in \mathbb{C}^{d}$. The point spectrum of the generator $\hat{A}$ is given by

$$
\sigma_{P}(\hat{A})=\left\{\lambda \in \mathbb{C}: \exists b \in \mathbb{C}^{d} \backslash\{0\}: e(\lambda) b \in \mathcal{B} \text { and } \lambda b-L(e(\lambda) b)=0\right\} .
$$

Let $\Lambda=\left\{\lambda_{1}, \ldots, \lambda_{p}\right\}$ be a finite set of eigenvalues of $\hat{A}$ such that $\operatorname{Re} \lambda_{j}>\beta$ for $j=1, \ldots, p$. For every $\lambda_{j}$ there exists a basis $\boldsymbol{\Phi}_{j}=\left(\Phi_{1}, \ldots, \Phi_{m_{j}}\right)$ of the generalized eigenspace $M\left(\lambda_{j}\right):=\operatorname{ker}\left(\left(\lambda_{j} \mathrm{Id}-\hat{A}\right)^{k_{j}}\right), k_{j} \in \mathbb{N}$. For such a basis there are matrices $B_{j} \in \mathbb{C}^{m_{j} \times m_{j}}$ with the single eigenvalue $\lambda_{j}$ and $\hat{A} \boldsymbol{\Phi}_{j}=\boldsymbol{\Phi}_{j} B_{j}$. Define $\boldsymbol{\Phi}_{\Lambda}=\left(\boldsymbol{\Phi}_{1}, \ldots, \boldsymbol{\Phi}_{p}\right), B_{\Lambda}=\operatorname{diag}\left(B_{1}, \ldots, B_{p}\right)$ and $m=m_{1}+\cdots+m_{p}$. For every vector $a \in \mathbb{C}^{m}$, the function $\hat{T}(t) \boldsymbol{\Phi}_{\Lambda} a$ with initial value $\boldsymbol{\Phi}_{\Lambda} a$ at $t=0$ may be defined on $\mathbb{R}$ by the relation

$$
\hat{T}(t) \boldsymbol{\Phi}_{\Lambda} a=\boldsymbol{\Phi}_{\Lambda} e^{B_{\Lambda} t} a, \quad \boldsymbol{\Phi}_{\Lambda}(u)=\boldsymbol{\Phi}_{\Lambda}(0) e^{B_{\Lambda} u}, u \leqslant 0 .
$$

Furthermore, there exists a $\hat{T}(t)$-invariant subspace $\hat{Q}_{\Lambda}$ of $\hat{\mathcal{B}}$ such that

$$
\hat{\mathcal{B}}=\hat{P}_{\Lambda} \oplus \hat{Q}_{\Lambda}
$$

where $\hat{P}_{\Lambda}=\left\{\hat{\varphi} \in \hat{\mathcal{B}}: \hat{\varphi}=\boldsymbol{\Phi}_{\Lambda} a\right.$ for some $\left.a \in \mathbb{C}^{m}\right\}$. In the special case of $\Lambda=$ $\Lambda(s):=\left\{\lambda \in \sigma_{P}(\hat{A}): \operatorname{Re} \lambda \geqslant s\right\}$ for some $s>\beta$ the set $\Lambda(s)$ is finite and one obtains for every $\varepsilon>0$ the estimate

$$
\|\hat{T}(t) \hat{\varphi}\|_{\hat{\mathcal{B}}} \leqslant k(\varepsilon) e^{\left(s^{\prime}+\varepsilon\right) t}\|\hat{\varphi}\|_{\hat{\mathcal{B}}} \quad \text { for } t \geqslant 0, \hat{\varphi} \in \hat{Q}_{\Lambda}
$$

where $s^{\prime}:=\max \left\{\sup \left\{\operatorname{Re} \lambda: \lambda \in \sigma_{P}(\hat{A}) \backslash \Lambda(s)\right\}, \beta\right\}$ and $k(\varepsilon)$ is a constant depending on $\varepsilon$. Let $\pi_{P}: \hat{\mathcal{B}} \rightarrow \hat{\mathcal{B}}$ be the projection onto $\hat{P}_{\Lambda}$ along the direct sum (4.2): $\pi_{P}(\hat{\varphi})=\hat{\varphi}^{P}$ if $\hat{\varphi}=\hat{\varphi}^{P}+\hat{\varphi}^{Q}, \hat{\varphi}^{P} \in \hat{P}_{\Lambda}, \hat{\varphi}^{Q} \in \hat{Q}_{\Lambda}$ and $\pi_{Q}=\operatorname{Id}-\pi_{P}$. Since the 
spaces $\hat{P}_{\Lambda}$ and $\hat{Q}_{\Lambda}$ are invariant under the operator $\hat{T}(t)$, the projections $\pi_{P}$ and $\pi_{Q}$ and their adjoints commute:

$$
\pi_{P}^{*} T^{*}(t)=T^{*}(t) \pi_{P}^{*} \quad \text { and } \quad \pi_{P}^{* *} T^{* *}(t)=T^{* *}(t) \pi_{P}^{* *} \quad \text { for every } t \geqslant 0,
$$

and analogously for the projection $\pi_{Q}$ onto $\hat{Q}_{\Lambda}$.

Furthermore, $\pi_{P}^{*} \mathcal{B}^{*}=\left(\hat{Q}_{\Lambda}\right)^{\perp}$, where $\left(\hat{Q}_{\Lambda}\right)^{\perp}$ denotes the annihilator of $\hat{Q}_{\Lambda}$, and there exists a basis $\boldsymbol{\Psi}_{\Lambda}=\left(\Psi_{1}, \ldots, \Psi_{m}\right)^{T}$ for $\pi_{P}^{*} \mathcal{B}^{*} \subseteq \mathcal{B}^{*}$ such that $\left\langle\Psi_{i}, \Phi_{j}\right\rangle=$ $\delta_{i j}$ for $i, j=1, \ldots, m$. One obtains

$$
\begin{aligned}
& \pi_{P} \hat{\varphi}=\sum_{i=1}^{m}\left\langle\Psi_{i}, \hat{\varphi}\right\rangle \Phi_{i}=\boldsymbol{\Phi}_{\Lambda}\left\langle\boldsymbol{\Psi}_{\Lambda}, \hat{\varphi}\right\rangle \quad \text { for all } \hat{\varphi} \in \hat{\mathcal{B}}, \\
& \pi_{P}^{* *} \varphi^{* *}=\sum_{i=1}^{m}\left\langle\Psi_{i}, \varphi^{* *}\right\rangle \Phi_{i}=\boldsymbol{\Phi}_{\Lambda}\left\langle\boldsymbol{\Psi}_{\Lambda}, \varphi^{* *}\right\rangle \quad \text { for all } \varphi^{* *} \in \mathcal{B}^{* *},
\end{aligned}
$$

using the notations $\left\langle\Psi_{\Lambda}, \hat{\varphi}\right\rangle:=\left(\left\langle\Psi_{1}, \hat{\varphi}\right\rangle, \ldots,\left\langle\Psi_{m}, \hat{\varphi}\right\rangle\right)^{T}$ and similarly for $\left\langle\Psi_{\Lambda}, \varphi^{* *}\right\rangle$.

For such a decomposition of the phase space $\hat{\mathcal{B}}$ into two subspaces with respect to a given subset of eigenvalues of the generator $\hat{A}$ we project the representation (3.6) of the solution onto these subspaces. We denote by $\mathbb{C}_{\beta}$ the set $\{z \in \mathbb{C}$ : $\operatorname{Re} z>\beta\}$.

Theorem 4.1. Let $\hat{\mathcal{B}}=\hat{P}_{\Lambda} \oplus \hat{Q}_{\Lambda}$ be a decomposition with respect to $\Lambda \subseteq \sigma_{P}(\hat{A}) \cap \mathbb{C}_{\beta}$. Then the solution $x=x(\cdot, \varphi, h)$ of (1.1) can be represented as

$$
\begin{gathered}
\pi_{P} \hat{x}_{t}=\hat{T}(t)\left(\pi_{P} \hat{\varphi}\right)+* \int_{0}^{t} T^{* *}(t-s)\left(\pi_{P}^{* *} \gamma\right) d h(s) \quad \text { for all } t \geqslant 0 \quad \text { in } \mathcal{B}^{* *}, \\
\pi_{Q} \hat{x}_{t}=\hat{T}(t)\left(\pi_{Q} \hat{\varphi}\right)+* \int_{0}^{t} T^{* *}(t-s)\left(\pi_{Q}^{* *} \gamma\right) d h(s) \quad \text { for all } t \geqslant 0 \quad \text { in } \mathcal{B}^{* *} .
\end{gathered}
$$

Proof. The proof follows easily from Theorem 3.5 and (3.4).

In the sequel we determine the asymptotic of the projections (4.7) and (4.8) to obtain the Lyapunov exponents. For the estimate of the projection (4.7) onto the finite dimensional subspace $\hat{P}_{\Lambda}$ we simplify this representation. The equations (4.5), (4.6), (4.1) and the definition (3.5) of $\gamma$ yield

$$
\begin{aligned}
& \hat{T}(t)\left(\pi_{P} \hat{\varphi}\right)=\hat{T}(t)\left(\mathbf{\Phi}_{\Lambda}\left\langle\mathbf{\Psi}_{\Lambda}, \hat{\varphi}\right\rangle\right)=\mathbf{\Phi}_{\Lambda} e^{B_{\Lambda} t}\left\langle\mathbf{\Psi}_{\Lambda}, \hat{\varphi}\right\rangle, \\
& T^{* *}(t-s)\left(\pi_{P}^{* *} \gamma\right)=T(t-s)\left(\mathbf{\Phi}_{\Lambda}\left\langle\mathbf{\Psi}_{\Lambda}, \gamma\right\rangle\right)=-\mathbf{\Phi}_{\Lambda} e^{B_{\Lambda}(t-s)} \widetilde{\mathbf{\Psi}}_{\Lambda}(0-) .
\end{aligned}
$$

Therefore we obtain by (4.7) the representation

$$
\begin{aligned}
\pi_{P} \hat{x}_{t}= & \boldsymbol{\Phi}_{\Lambda} U_{\Lambda}(t) \\
& \text { with } U_{\Lambda}(t):=\left(e^{B_{\Lambda} t}\left\langle\mathbf{\Psi}_{\Lambda}, \hat{\varphi}\right\rangle-\int_{0}^{t} e^{B_{\Lambda}(t-s)} \widetilde{\mathbf{\Psi}}_{\Lambda}(0-) d h(s)\right) .
\end{aligned}
$$

The function $U_{\Lambda}:[0, \infty) \rightarrow \mathbb{C}^{m}$ is called the coordinate process as it represents the coordinate of the projection $\pi_{p} \hat{x}_{t}$ with respect to the basis $\boldsymbol{\Phi}_{\Lambda}$ for a fixed 
$t \geqslant 0$. Note that the coordinate process $U_{\Lambda}$ is the solution of an ordinary integral equation in $\mathbb{C}^{m}$ :

$$
u(t)=\left\langle\Psi_{\Lambda}, \hat{\varphi}\right\rangle+\int_{0}^{t} B_{\Lambda} u(s) d s-\widetilde{\Psi}_{\Lambda}(0-) h(t), \quad t \geqslant 0 .
$$
obtains

Due to the representation (4.10) and the finite dimensionality of $\hat{P}_{\Lambda}$ one

$$
\limsup _{t \rightarrow \infty} \frac{1}{t} \log \left\|\pi_{P} \hat{x}_{t}\right\|_{\hat{\mathcal{B}}}=\limsup _{t \rightarrow \infty} \frac{1}{t} \log \left|U_{\Lambda}(t)\right| .
$$

Therefore the asymptotic of $\left\|\pi_{P} \hat{x}_{t}\right\|_{\mathcal{B}}$ can be determined by the one of the solution of the ordinary integral equation (4.11). This calculation has been already accomplished in [9] to which we refer in the following result.

Let $\hat{\mathcal{B}}=\hat{P}_{\Lambda} \oplus \hat{Q}_{\Lambda}$ be a decomposition with respect to $\Lambda \subseteq\left\{\lambda \in \sigma_{P}(\hat{A})\right.$ : $\operatorname{Re} \lambda \geqslant v\}$ with $v>\beta$. Assume $h(t)=\mathcal{O}\left(e^{\kappa t}\right)$ as $t \rightarrow \infty$ for a constant $\kappa<v$ where $\mathcal{O}$ denotes the Landau symbol. Then for $t \geqslant 0$ define

$$
Y_{\Lambda}(t):=\lim _{T \rightarrow \infty} \int_{t}^{T} e^{B_{\Lambda}(t-s)} \widetilde{\mathbf{\Psi}}_{\Lambda}(0-) d h(s),
$$

where the integral is defined according to (3.1). Since $\sigma\left(B_{\Lambda}\right)=\Lambda$ and $\kappa<v$ the limit exists.

Theorem 4.2. Let $\hat{\mathcal{B}}=\hat{P}_{\Lambda_{i}} \oplus \hat{Q}_{\Lambda_{i}}$ be the decompositions with respect to $\Lambda_{i}:=\{\lambda \in$ $\left.\sigma_{P}(\hat{A}): \operatorname{Re} \lambda=v_{i}\right\}$ with $v_{1}>\cdots>v_{p}>\beta$ for $i=1, \ldots, p$ and let $\boldsymbol{\Psi}_{i}$ be the basis of $\left(\hat{Q}_{\Lambda_{i}}\right)^{\perp}$ as chosen above. Assume $h(t)=\mathcal{O}\left(e^{\kappa t}\right)$ as $t \rightarrow \infty$ for a constant $\kappa<v_{p}$.

1) If $\left\langle\boldsymbol{\Psi}_{i}, \hat{\varphi}\right\rangle=Y_{\Lambda_{i}}(0)$ for $i=1, \ldots, l-1, l \in\{1, \ldots, p\}$ and $\left\langle\boldsymbol{\Psi}_{l}, \hat{\varphi}\right\rangle \neq Y_{\Lambda_{l}}(0)$ then the solution $x=x(\cdot, \varphi, h)$ of (1.1) for $\varphi \in \mathcal{B}$ obeys

$$
\lim _{t \rightarrow \infty} \frac{1}{t} \log \left\|\pi_{P} \hat{x}_{t}\right\|_{\hat{\mathcal{B}}}=v_{l}
$$

2) If $\left\langle\Psi_{i}, \hat{\varphi}\right\rangle=Y_{\Lambda_{i}}(0)$ for $i=1, \ldots, p$ then the solution $x=x(\cdot, \varphi, h)$ of $(1.1)$ for $\varphi \in \mathcal{B}$ obeys

$$
\limsup _{t \rightarrow \infty} \frac{1}{t} \log \left\|\pi_{P} \hat{x}_{t}\right\|_{\hat{\mathcal{B}}} \leqslant \kappa .
$$

Here $\pi_{P}$ denotes the projection onto $\hat{P}_{\Lambda}$ if the phase space is decomposed according to $\hat{\mathcal{B}}=\hat{P}_{\Lambda} \oplus \hat{Q}_{\Lambda}$ with respect to $\Lambda:=\Lambda_{1} \cup \cdots \cup \Lambda_{p}$.

Proof. Similar to Theorem 8 in [9].

To establish an upper bound for the projection $\pi_{Q} \hat{x}_{t}$ of the solution $x=$ $x(\cdot, \varphi, h)$ onto the complementary subspace $\hat{Q}_{\Lambda}$ we use the representation (4.8). Determining the asymptotic behavior of the weak*-integral in the representation (4.8) will result in estimating the total variation of the solution of the adjoint equation (2.5), but only with forcing functions in the subspace $\left(\pi_{Q}^{*}\left(\mathcal{B}^{*}\right)\right)^{\sim}$. 
Lemma 4.3. Let $\hat{\mathcal{B}}=\hat{P}_{\Lambda} \oplus \hat{Q}_{\Lambda}$ be a decomposition with respect to $\Lambda=\left\{\lambda \in \sigma_{P}(\hat{A})\right.$ : $\operatorname{Re} \lambda \geqslant s\}$ for some $s>\beta$ and let $\pi_{Q}$ be the according projection onto $\hat{Q}_{\Lambda}$. Then for every $\varepsilon>0$ there exist some constants $k_{0}=k_{0}(\varepsilon)$ and $k_{1}=k_{1}(\varepsilon)$, such that the solution $y\left(\cdot,\left[\pi^{*} \psi\right]^{\sim}\right)$ of equation (2.5) for $\psi \in \mathcal{B}^{*}$ satisfies the following estimate for $t \geqslant 0$ :

$$
\operatorname{Var}\left(y\left(\cdot,\left[\pi_{Q}^{*} \psi\right] \tilde{)}\right),[-t, 0]\right) \leqslant\left(k_{0} N(t)+k_{1} \int_{0}^{t} N(t-u) e^{\left(s^{\prime}+\varepsilon\right) u} d u\right)\|\psi\|_{\mathcal{B}^{*}},
$$

where $s^{\prime}:=\max \left\{\sup \left\{\operatorname{Re} \lambda: \lambda \in \sigma_{P}(\hat{A}) \backslash \Lambda\right\}, \beta\right\}$.

Proof. We denote by $c$ a generic constant only depending on $\varepsilon$. The equations (2.10), (2.9) and the inequality (4.3) imply for the solution $y\left(\cdot,\left[\pi_{Q}^{*} \psi\right]^{\sim}\right)$ of the adjoint equation (2.5) for $\psi \in \mathcal{B}^{*}, t>0$ and $\delta>0$

$$
\begin{aligned}
& \mid y\left(-t,\left[\pi_{Q}^{*} \psi \widetilde{]}\right)|=|\left[T^{*}(t)\left(\pi_{Q}^{*} \psi\right) \widetilde{{ }^{(}(0-)} \mid\right.\right. \\
& \leqslant \operatorname{Var}\left(\left[T^{*}(t)\left(\pi_{Q}^{*} \psi\right)\right]^{\sim}(\cdot),[-\delta, 0]\right) \\
& \leqslant c N(\delta)\left\|T^{*}(t)\left(\pi_{Q}^{*} \psi\right)\right\|_{\mathcal{B}^{*}} \\
& \leqslant c N(\delta)\|\psi\|_{\mathcal{B}^{*}}\left\|T^{*}(t) \pi_{Q}^{*}\right\|_{\mathcal{B}^{*} \rightarrow \mathcal{B}^{*}} \\
& =c N(\delta)\|\psi\|_{\mathcal{B}^{*}}\left\|\hat{T}(t) \pi_{Q}\right\|_{\hat{\mathcal{B}} \rightarrow \hat{\mathcal{B}}} \\
& \leqslant c N(\delta)\|\psi\|_{\mathcal{B}^{*}} e^{\left(s^{\prime}+\varepsilon\right) t} .
\end{aligned}
$$

Since $\delta>0$ is arbitrary this yields

$$
\left|y\left(-t,\left[\pi_{Q}^{*} \psi\right]^{\sim}\right)\right| \leqslant c N(0)\|\psi\|_{\mathcal{B}^{*}} e^{\left(s^{\prime}+\varepsilon\right) t} .
$$

For $y=y\left(\cdot,\left[\pi_{Q}^{*} \psi\right]^{\sim}\right)$ define

$$
F(s):=\int_{s}^{0} y(u) \mu(s-u) d u, \quad s \leqslant 0 .
$$

If we set $\mu(u)=0$ for $u>0$ we obtain for $t \geqslant 0$

$$
F(s)=\int_{-t}^{0} y(u) \mu(s-u) d u \quad \text { for } s \in[-t, 0] .
$$

We infer from (2.3) and (4.13)

$$
\begin{aligned}
\operatorname{Var}(F,[-t, 0]) & \leqslant \int_{-t}^{0}|y(u)| \operatorname{Var}(\mu,[-t-u, 0]) d u \\
& \leqslant c \int_{-t}^{0} N(0)\|\psi\|_{\mathcal{B}^{*}} e^{-\left(s^{\prime}+\varepsilon\right) u}\|L\|_{\mathcal{B} \rightarrow \mathbb{C}^{d}} N(t+u) M(0) d u \\
& \leqslant c\|\psi\|_{\mathcal{B}^{*}} \int_{0}^{t} N(t-u) e^{\left(s^{\prime}+\varepsilon\right) u} d u
\end{aligned}
$$


From (2.9) we obtain for every $\psi \in \mathcal{B}^{*}$ and $t \geqslant 0$

$$
\operatorname{Var}\left(\left[\pi_{Q}^{*} \psi\right]^{\tilde{y}},[-t, 0]\right) \leqslant c N(t)\left\|\pi_{Q}^{*} \psi\right\|_{\mathcal{B}^{*}} \leqslant c N(t)\left\|\pi_{Q}\right\|_{\mathcal{B} \rightarrow \mathcal{B}}\|\psi\|_{\mathcal{B}^{*}} .
$$

Since $y$ obeys the adjoint equation (2.5) we have

$$
\operatorname{Var}\left(y\left(\cdot,\left[\pi_{Q}^{*} \psi\right]^{\sim}\right),[-t, 0]\right) \leqslant \operatorname{Var}(F,[-t, 0])+\operatorname{Var}\left(\left[\pi_{Q}^{*} \psi\right]^{\sim},[-t, 0]\right) .
$$

Therefore the application of inequalities (4.14) and (4.15) finishes the proof.

Lemma 4.3 provides an upper bound of the total variation of the solution of the adjoint equation (2.5) for some forcing functions subject to the decomposition $\hat{\mathcal{B}}=\hat{P}_{\Lambda} \oplus \hat{Q}_{\Lambda}$ of the phase space with respect to a spectral set $\Lambda$. This connection of the solution of the adjoint equation and the decomposition enables us to estimate the asymptotic of the projection $\pi_{Q} \hat{x}_{t}$ of the solution $x$ of equation (1.1).

Theorem 4.4. Let $\hat{\mathcal{B}}=\hat{P}_{\Lambda} \oplus \hat{Q}_{\Lambda}$ be a decomposition with respect to $\Lambda=\{\lambda \in$ $\left.\sigma_{P}(\hat{A}): \operatorname{Re} \lambda \geqslant s\right\}$ for some $s>\beta$ and let $\pi_{Q}$ be the according projection onto $\hat{Q}_{\Lambda}$. Then for every $\varepsilon>0$ there exists a constant $k=k(\varepsilon)$, such that the history of the solution $x=x(\cdot, \varphi, h)$ of equation (1.1) for $\varphi \in \mathcal{B}$ satisfies

$$
\left\|\pi_{Q} \hat{x}_{t}\right\|_{\hat{\mathcal{B}}} \leqslant k\left(\|\varphi\|_{\mathcal{B}} e^{\left(s^{\prime}+\varepsilon\right) t}+\|h\|_{C[0, t]}\left(N(t)+\int_{0}^{t} N(t-u) e^{\left(s^{\prime}+\varepsilon\right) u} d u\right)\right)
$$

for every $t \geqslant 0$ with $s^{\prime}:=\max \left\{\sup \left\{\operatorname{Re} \lambda: \lambda \in \sigma_{P}(\hat{A}) \backslash \Lambda\right\}, \beta\right\}$.

Proof. Theorem 4.1 implies

$$
\left\|\pi_{Q} \hat{x}_{t}\right\|_{\hat{\mathcal{B}}} \leqslant\left\|\hat{T}(t)\left(\pi_{Q} \hat{\varphi}\right)\right\|_{\hat{\mathcal{B}}}+\left\|* \int_{0}^{t} T^{* *}(t-s)\left(\pi_{Q}^{* *} \gamma\right) d h(s)\right\|_{\hat{\mathcal{B}}} .
$$

By use of the definition (3.5) of $\gamma$, relation (2.10) and Lemma 4.3 the second term in $(4.16)$ can be estimated by

$$
\begin{aligned}
\| \int_{0}^{t} & T^{* *}(t-s)\left(\pi_{Q}^{* *} \gamma\right) d h(s) \|_{\hat{\mathcal{B}}} \\
= & \sup _{\|\psi\|_{\mathcal{B}^{*} \leqslant 1}}\left|\left\langle\psi, * \int_{0}^{t} T^{* *}(t-s)\left(\pi_{Q}^{* *} \gamma\right) d h(s)\right\rangle\right| \\
= & \sup _{\|\psi\|_{\mathcal{B}^{*}} \leqslant 1}\left|\int_{0}^{t}\left\langle\psi, T^{* *}(t-s)\left(\pi_{Q}^{* *} \gamma\right)\right\rangle d h(s)\right| \\
= & \sup _{\|\psi\|_{\mathcal{B}^{*}} \leqslant 1}\left|\int_{0}^{t}\left\langle T^{*}(t-s)\left(\pi_{Q}^{*} \psi\right), \gamma\right\rangle d h(s)\right| \\
= & \sup _{\|\psi\|_{\mathcal{B}^{*} \leqslant 1}}\left|-\int_{0}^{t}\left[T^{*}(t-s)\left(\pi_{Q}^{*} \psi\right)\right]^{\sim}(0-) d h(s)\right| \\
= & \sup _{\|\psi\|_{\mathcal{B}^{*} \leqslant 1}}\left|-\int_{0}^{t} y\left(s-t,\left[\pi_{Q}^{*} \psi\right]^{\tilde{)}}\right) d h(s)\right|
\end{aligned}
$$




$$
\begin{aligned}
& \leqslant 2\|h\|_{C[0, t]} \sup _{\|\psi\|_{\mathcal{B}^{*}} \leqslant 1}\left\{\operatorname{Var}\left(y\left(\cdot,\left[\pi_{Q}^{*} \psi\right]^{\tilde{T}}\right),[-t, 0]\right)\right\} \\
& \leqslant 2 c\|h\|_{C[0, t]}\left(N(t)+\int_{0}^{t} N(t-u) e^{\left(s^{\prime}+\varepsilon\right) u} d u\right)
\end{aligned}
$$

with a constant $c=c(\varepsilon)>0$. Applying (4.3) for estimating the first term in (4.16) finishes the proof.

The estimates of the two projections in this section enable us in the next theorem to determine the asymptotic of the solution $x(\cdot, \varphi, h)$ of equation $(1.1)$ with respect to the initial condition $\varphi \in \mathcal{B}$. For that we decompose the phase space $\mathcal{B}$ with respect to $\Lambda(s)=\{\lambda \in \sigma(\hat{A}): \operatorname{Re} \lambda \geqslant s\}$ for $s>\beta$ where we assume

$$
\begin{aligned}
& \Lambda(s)=\Lambda_{1} \cup \cdots \cup \Lambda_{p}, \quad p \in \mathbb{N}, \\
& \Lambda_{i}=\left\{\lambda \in \sigma_{P}(\hat{A}): \operatorname{Re} \lambda=v_{i}\right\} \quad \text { for some } v_{1}>\cdots>v_{p} \geqslant s .
\end{aligned}
$$

Recall that $\Lambda(s)$ is finite and therefore the decomposition in (4.17) is not a restriction. As before we assume that the space $\mathcal{B}$ satisfies the Conditions (A), (B) and (C). As in Theorem 4.2 , the vector $\boldsymbol{\Psi}_{i}$ denotes the basis for $\left(\hat{Q}_{\Lambda_{i}}\right)^{\perp}$ with respect to the decomposition $\hat{\mathcal{B}}=\hat{P}_{\Lambda_{i}} \oplus \hat{Q}_{\Lambda_{i}}$.

Theorem 4.5. Let $\Lambda=\Lambda(s)$ be given as in (4.17) for a constant $s>\max \{\beta, 0\}$. Assume that there exist constants $\kappa, \theta \in[0, s)$, such that for every $\varepsilon>0$ the conditions

$$
\begin{aligned}
& \|h(u)\|_{C[0, t]}=\mathcal{O}\left(e^{(\kappa+\varepsilon) t}\right) \quad \text { for } t \rightarrow \infty, \\
& N(t)+\int_{0}^{t} N(t-u) e^{\left(s^{\prime}+\varepsilon\right) u} d u=\mathcal{O}\left(e^{(\theta-\kappa+\varepsilon) t}\right) \quad \text { for } t \rightarrow \infty
\end{aligned}
$$

are satisfied with $s^{\prime}:=\max \left\{\sup \left\{\operatorname{Re} \lambda: \lambda \in \sigma_{P}(\hat{A}) \backslash \Lambda\right\}, \beta\right\}$.

1) If $\left\langle\boldsymbol{\Psi}_{i}, \hat{\varphi}\right\rangle=Y_{\Lambda_{i}}(0)$ for $i=1, \ldots, l-1, l \in\{1, \ldots, p\}$, and $\left\langle\boldsymbol{\Psi}_{l}, \hat{\varphi}\right\rangle \neq Y_{\Lambda_{l}}(0)$ then the solution $x=x(\cdot, \varphi, h)$ of (1.1) for $\varphi \in \mathcal{B}$ obeys

$$
\lim _{t \rightarrow \infty} \frac{\log \left\|\hat{x}_{t}\right\|_{\hat{\mathcal{B}}}}{t}=v_{l}
$$

2) If $\left\langle\boldsymbol{\Psi}_{i}, \hat{\varphi}\right\rangle=Y_{\Lambda_{i}}(0)$ for $i=1, \ldots, p$ then the solution $x=x(\cdot, \varphi, h)$ of (1.1) for $\varphi \in \mathcal{B}$ obeys

$$
\limsup _{t \rightarrow \infty} \frac{\log \left\|\hat{x}_{t}\right\|_{\hat{\mathcal{B}}}}{t} \leqslant \max \left\{\kappa, s^{\prime}, \theta\right\} .
$$

Proof. Theorem 4.4 implies

$$
\limsup _{t \rightarrow \infty} \frac{1}{t} \log \left\|\pi_{Q} \hat{x}_{t}\right\|_{\hat{\mathcal{B}}} \leqslant \max \left\{s^{\prime}, \theta\right\} .
$$

If $\hat{\varphi}$ satisfies the assumptions under 1 ), then Theorem 4.2 yields

$$
\lim _{t \rightarrow \infty} \frac{1}{t} \log \left\|\pi_{P} \hat{x}_{t}\right\|_{\hat{\mathcal{B}}}=v_{l} .
$$


Therefore, one obtains

$$
\begin{aligned}
\lim _{t \rightarrow \infty} \frac{1}{t} \log \left\|\hat{x}_{t}\right\|_{\hat{\mathcal{B}}} & =\lim _{t \rightarrow \infty} \frac{1}{t} \log \left\|\pi_{P} \hat{x}_{t}+\pi_{Q} \hat{x}_{t}\right\|_{\hat{\mathcal{B}}} \\
& =\lim _{t \rightarrow \infty} \frac{1}{t} \log \left(\left\|\pi_{P} \hat{x}_{t}\right\|_{\hat{\mathcal{B}}}|1+o(1)|\right) \\
& =v_{l} .
\end{aligned}
$$

If the assumptions under 2) hold we have

$$
\limsup _{t \rightarrow \infty} \frac{1}{t} \log \left\|\pi_{P} \hat{x}_{t}\right\|_{\hat{\mathcal{B}}} \leqslant \kappa
$$

according to Theorem 4.2 which finishes the proof in combination with (4.20).

\section{The example of a stochastic equation}

In this section we apply Theorem 4.5 to a stochastic differential equation with infinite delay. For example, such equations are encountered in the linear theory of viscoelasticity if the equation of motion is perturbed by an additive noise. For a description of such models see [7]. For literature on stochastic functional differential equations we refer to [6] and [8].

Although we replace the complex space $\mathbb{C}^{d}$ by $\mathbb{R}^{d}$ in the following example the theory and results of the previous sections can be applied due to an appropriate complexification of the underlying spaces, see III.7 in [1]. In particular we consider phase spaces $\mathcal{B}\left(\mathbb{R}_{-}, \mathbb{R}^{d}\right) \subseteq\left\{\varphi:(-\infty, 0] \rightarrow \mathbb{R}^{d}\right\}$ satisfying the Condition (A) with an obvious modification.

Let $(\Omega, P, \mathcal{F})$ be a probability space with filtration $\left\{\mathcal{F}_{t}\right\}_{t \geqslant 0}$ and $\{W(t, \omega): t \geqslant$ $0, \omega \in \Omega\}$ be an $\mathbb{R}^{d}$-valued Wiener process on this space. We assume on the phase space $\mathcal{B}=\mathcal{B}\left(\mathbb{R}_{-}, \mathbb{R}^{d}\right)$ the Borel $\sigma$-field generated by the semi-norm $\|\cdot\|_{\mathcal{B}}$. The initial condition is a $\mathcal{B}$-valued $\mathcal{F}_{0}$-measurable random variable $\Phi(\cdot)=\{\Phi(u, \cdot): u \leqslant 0\}$ on $(\Omega, P, \mathcal{F})$. We consider the stochastic differential equation

$$
\begin{aligned}
& X(t, \omega)=\Phi(0, \omega)+\int_{0}^{t} L\left(X_{s}(\cdot, \omega)\right) d s+W(t, \omega) \quad \text { for } t \geqslant 0, \\
& X(u, \omega)=\Phi(u, \omega) \quad \text { for } u \leqslant 0
\end{aligned}
$$

where $L: \mathcal{B} \rightarrow \mathbb{R}^{d}$ is a linear continuous operator.

A solution of equation (5.1) is a stochastic process $\{X(t, \cdot): t \in \mathbb{R}\}$ satisfying both relations in (5.1) P-a.s. and where every $X(t, \cdot)$ is $\mathcal{F}_{t}$-measurable. It is immediate that one can consider the initial value problem (5.1) for fixed $\omega \in \Omega$ as the deterministic equation (1.1). Hence, there exists a solution $\{X(t, \cdot): t \in \mathbb{R}\}$ of equation (5.1) on the set $\Omega^{\prime} \subseteq \Omega$ with $P\left(\Omega^{\prime}\right)=1$ where $W(\cdot, \omega)$ is continuous on $[0, \infty)$ for every $\omega \in \Omega^{\prime}$. The required measurability of the solution follows by its representation $(3.3)$, see [11]. 
Corollary 5.1. Let $\mathcal{B}$ satisfy the Conditions (A), $(B)$ and $(C)$ with a bounded function $N$ and assume $\beta<0$. If $v:=\max \left\{\operatorname{Re} \lambda: \lambda \in \sigma_{P}(\hat{A})\right\}>0$ then for every initial process $\Phi(\cdot)$ the solution $\{X(t, \cdot): t \in \mathbb{R}\}$ of equation (5.1) obeys

$$
\lim _{t \rightarrow \infty} \frac{1}{t} \log \left\|\hat{X}_{t}(\cdot, \omega)\right\|_{\hat{\mathcal{B}}}=v \quad \text { for P-almost all } \omega \in \Omega \text {. }
$$

Proof. We apply Theorem 4.5 to the finite spectral set $\Lambda=\{\lambda \in \sigma(\hat{A}): \operatorname{Re} \lambda>0\}$. By the law of iterated logarithm we have P-a.s. $W(t, \cdot)=\mathcal{O}\left(e^{\varepsilon t}\right)$ as $t \rightarrow \infty$ for every $\varepsilon>0$. Since $s^{\prime} \leqslant 0$ the conditions (4.18) and (4.19) are fulfilled with $\kappa=\theta=0$. Therefore it remains to show that condition 1) in Theorem 4.5 is satisfied P-a.s. for $l=1$ that is $\left\langle\boldsymbol{\Psi}_{1}, \hat{\Phi}(\cdot)\right\rangle \neq Y_{\Lambda_{1}}(0, \cdot)$ P-a.s., where

$$
Y_{\Lambda_{1}}(0, \cdot)=\lim _{T \rightarrow \infty} \int_{0}^{T} e^{-B_{\Lambda_{1}} s} \widetilde{\boldsymbol{\Psi}}_{1}(0-) d W(s, \cdot)
$$

and the integral in (5.2) is defined for every $\omega \in \Omega^{\prime}$ as in (4.12).

For every $T>0$ the integral in (5.2) is a Gaussian random variable independent of the $\sigma$-field $\mathcal{F}_{0}$ by the definition of a Wiener process. Therefore, $Y_{\Lambda_{1}}(0, \cdot)=\left(Y_{\Lambda_{1}, 1}(0, \cdot), \ldots, Y_{\Lambda_{1}, m}(0, \cdot)\right)^{T}$ is also a Gaussian random variable independent of the $\sigma$-field $\mathcal{F}_{0}$.

Firstly, assume that there exists a component $Y_{\Lambda_{1}, i_{0}}(0, \cdot)$ which is a nondegenerate Gaussian random variable with Lebesgue density. This results in

$$
P\left(\left\langle\Psi_{1}, \hat{\Phi}(\cdot)\right\rangle=Y_{\Lambda_{1}}(0, \cdot)\right) \leqslant P\left(\left\langle\Psi_{1, i_{0}}, \hat{\Phi}(\cdot)\right\rangle=Y_{\Lambda_{1, i_{0}}}(0, \cdot)\right)=0,
$$

where $\boldsymbol{\Psi}_{1}=\left(\Psi_{1,1}, \ldots, \Psi_{1, m}\right)^{T}$.

Secondly, assume for a contradiction, that every component of $Y_{\Lambda_{1}}(0, \cdot)$ is a degenerate Gaussian random variable. Then, we have $Y_{\Lambda_{1}}(0, \cdot)=0$ P-a.s. which yields $\widetilde{\boldsymbol{\Psi}}_{1}(0-)=0$ by Itô's isometry. Denoting by $\pi_{P_{1}}$ the projection onto $P_{\Lambda_{1}}$ we derive for every $\psi \in \pi_{P_{1}}^{*} \mathcal{B}^{*}$ by $(4.9),(4.4)$ and $(2.10)$ :

$$
0=\left\langle\psi, T^{* *}(t)\left(\pi_{P_{1}}^{* *} \gamma\right)\right\rangle=\left\langle T^{*}(t) \psi, \gamma\right\rangle=-y(-t, \widetilde{\psi}) \quad \text { for every } t \geqslant 0,
$$

where $y(\cdot, \widetilde{\psi})$ denotes the solution of the adjoint equation (2.5). But zero is a solution of the adjoint equation if and only if the forcing function is zero. Hence, we obtain $\widetilde{\psi}=0$ for every $\psi \in \pi_{P_{1}}^{*} \mathcal{B}^{*}$.

Now, we show that the representation (2.8) remains true for every $\varphi \in P_{\Lambda_{1}}$ :

$$
\langle\zeta, \varphi\rangle=\int d \widetilde{\zeta}(u) \varphi(u) \quad \text { for every } \zeta \in \mathcal{B}^{*}
$$

Then, we have $\langle\psi, \varphi\rangle=0$ for every $\varphi \in P_{\Lambda_{1}}$ and $\psi \in \pi_{P_{1}}^{*} \mathcal{B}^{*}$, since $\widetilde{\psi}=0$. But this contradicts the projection (4.5) onto $P_{\Lambda_{1}}$.

To establish (5.3), we approximate $\varphi \in P_{\Lambda_{1}}$ by functions $\theta^{m} \in C_{c}\left(\mathbb{R}_{-}, \mathbb{C}^{d}\right)$ which equal $\varphi$ on $[-m+1,0]$, are zero on $(-\infty,-m]$ and are linear on $[-m,-m+1]$. 
Since $\operatorname{Re} \lambda=v>0$ for $\lambda \in \Lambda_{1}$ we have $|\varphi(u)| \rightarrow 0$ for $u \rightarrow-\infty$. Therefore, by use of the estimate

$$
\left\|\theta^{m}-\theta^{n}\right\|_{\mathcal{B}} \leqslant\left(\sup _{s \geqslant 0}|N(s)|\right)\left(\sup _{u \leqslant 0}\left|\theta^{m}(u)-\theta^{n}(u)\right|\right),
$$

which is a consequence of (A.3), Condition (C) implies $\left\|\theta^{m}-\varphi\right\|_{\mathcal{B}} \rightarrow 0$ for $m \rightarrow \infty$. On the other hand, the functions $\theta^{m} \in C_{c}\left(\mathbb{R}_{-}, \mathbb{C}^{d}\right)$ obey by $(2.8)$ :

$$
\left\langle\zeta, \theta^{m}\right\rangle=\int_{(-m+1,0]} d \widetilde{\zeta}(u) \varphi(u)+\int_{[-m,-m+1]} d \widetilde{\zeta}(u) \theta^{m}(u) .
$$

Since $\operatorname{Var}(\widetilde{\zeta},(-\infty, 0])$ is finite by $(2.9)$, the first integral converges to the integral in (5.3) and the second one to zero for $m \rightarrow \infty$.

Remark 5.2. For the example $C_{\gamma}\left(\mathbb{R}_{-}, \mathbb{R}^{d}\right)$ of a phase space in section 1 the function $N$ in Condition (A) is bounded and $\beta$ is negative if $\gamma<0$. The next example $\left(\mathbb{R}^{d} \times L_{g}^{p}\right)\left(\mathbb{R}_{-}, \mathbb{R}^{d}\right)$ in section 1 satisfies the conditions on the space $\mathcal{B}$ in Corollary 5.1 if ess $\sup \{g(u-t) / g(u): u \leqslant 0\} \rightarrow 0$ as $t \rightarrow \infty$ where we assume for simplicity $g(u)>0$ for Lebesgue almost all $u \leqslant 0$. These results are due to the fact that in these cases the spaces are so-called uniform fading memory spaces. For details see [5] or [11].

\section{References}

[1] O. Diekmann, S. A. van Gils, S. M. Verduyn Lunel, and H.-O. Walther. Delay equations. Functional-, complex-, and nonlinear analysis. Springer-Verlag New York, 1995.

[2] R. E. Edwards. Functional analysis. Theory and applications. Holt Rinehart and Winston, New York, 1965.

[3] J. K. Hale and J. Kato. Phase space for retarded equations with infinite delay. Funkc. Ekvacioj, Ser. Int., 21:11-41, 1978.

[4] J. K. Hale and S. M. Verduyn Lunel. Introduction to functional differential equations. Springer New York, 1993.

[5] Y. Hino, S. Murakami, and T. Naito. Functional differential equations with infinite delay. Lecture Notes in Mathematics, 1473. Springer Berlin, 1991.

[6] X. Mao. Stochastic differential equations and their applications. Horwood Publishing, Chichester, 1997.

[7] V. J. Mizel and V. Trutzer. Stochastic hereditary equations: Existence and asymptotic stability. J. Integral Equations, 7:1-72, 1984.

[8] S.-E. A. Mohammed. Stochastic functional differential equations. Pitman, Boston, 1984.

[9] S.-E. A. Mohammed and M. K. R. Scheutzow. Lyapunov exponents and stationary solutions for affine stochastic delay equations. Stochastics Stochastics Rep., 29(2):259-283, 1990. 
[10] S.-E. A. Mohammed, M. K. R. Scheutzow, and H. von Weizsäcker. Hyperbolic state space decomposition for a linear stochastic delay equation. SIAM J. Control Optimization, 24:543-551, 1986.

[11] M. Riedle. Stochastische Differentialgleichungen mit unendlichem Gedächtnis (Stochastic differential equations with infinite delay). $\mathrm{PhD}$ thesis, Humboldt University of Berlin, Berlin, 2003.

Markus Riedle

Institute of Mathematics

Humboldt University of Berlin

Unter den Linden 6

D-10099 Berlin

Germany

e-mail: riedle@mathematik.hu-berlin.de

Submitted: September 3, 2003

Revised: August 15, 2004

(D) To access this journal online:

(4D) http://www.birkhauser.ch 